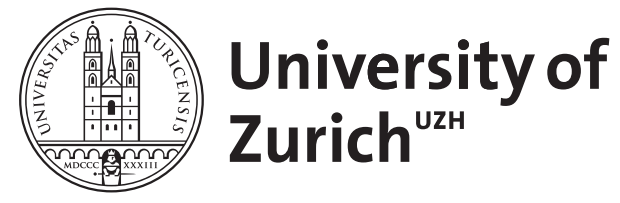
Archive

University of Zurich

University Library

Strickhofstrasse 39

CH-8057 Zurich

www.zora.uzh.ch

Year: 1996

\title{
Erfassung der Magenfunktionalität mittels MRI
}

Kunz, P ; Feinle, C ; Schwizer, W ; Fried, M ; Boesiger, P

DOI: https://doi.org/10.1515/bmte.1996.41.s1.140

Posted at the Zurich Open Repository and Archive, University of Zurich

ZORA URL: https://doi.org/10.5167/uzh-155379

Journal Article

Published Version

Originally published at:

Kunz, P; Feinle, C; Schwizer, W; Fried, M; Boesiger, P (1996). Erfassung der Magenfunktionalität mittels MRI. Biomedizinische Technik. Biomedical engineering, 41(s1):140-141.

DOI: https://doi.org/10.1515/bmte.1996.41.s1.140 


\title{
Erfassung der Magenfunktionalität mittels MRI
}

\author{
P. Kunz ${ }^{1}$, C. Feinle ${ }^{2}$, W. Schwizer ${ }^{2}$, M. Fried ${ }^{2}$, P. Boesiger ${ }^{1}$ \\ 'Institut für Biomedizinische Technik und Medizinische Informatik, Universität und ETH Zürich \\ ${ }^{2}$ Abteilung Gastroenterologie, Universitätsspital Zürich, Schweiz.
}

\section{EINLEITUNG}

Die genauen motorischen Abläufe des Magens, mit denen die Entleerung von Mahlzeiten aus dem Magen gesteuert wird, sind bisher noch nicht vollständig untersucht und erklärt worden. Mit einer bereits validierten Methode zur Messung der Magenentleerung mittels bildgebender Magnetresonanz [1,2] war es bisher möglich, die Magenentleerung flüssiger Testmahlzeit zu untersuchen. Mit den Ergebnissen konnten wir zeigen, dass im Gegensatz zur vorherrschenden Meinung das Antrum eine wichtige Rolle bei der Entleerung von flüssigen Mahlzeiten spielt.

Um aber die zugrundeliegenden Mechanismen genauer untersuchen zu können, ist es unumgänglich, die Magenentleerung fester und flüssiger Mahlzeiten zu erfassen. Unser Ziel bestand aus diesem Grund in der Entwicklung einer physiologischen festen Testmahlzeit und eines Messprotokolls zur Erfassung der Magenentleerung und der -motilität mittels bildgebender Magnetresonanz. Dadurch kann die Magenentleerung und die Magenmotilität der festen Testmahlzeit mit einer flüssigen Testmahlzeit verglichen werden, welche eine identische Nährstoffzusammensetzung und denselben Energiegehalt besitzen.

\section{METHODEN}

Probandenkollektiv und Testmahlzeiten: Magenentleerung und -motilität wurden bei 5 Probanden (Alter: 23 - 42 Jahre) zweimal an zwei verschiedenen Tagen gemessen. Bei einer Messung wurde die flüssige und bei der anderen die feste Testmahlzeit verabreicht. Tabelle 1 zeigt die Zusammensetzung der beiden Testmahlzeiten.

Tabelle 1: Zusammensetzung der beiden Mahlzeiten

\begin{tabular}{c|c} 
Feste Mahlzeit: & Flüssige Mahlzeit: \\
\hline $108 \mathrm{~g} \mathrm{Ei}$ & $154 \mathrm{ml}$ Carbospare \\
44 g Speck & $264 \mathrm{ml}$ Intralipid ${ }^{\otimes} 10 \%$ \\
$98 \mathrm{~g} \mathrm{Kartoffeln}$ & $10 \mathrm{~g}$ Albumin \\
$5 \mathrm{~g}$ Butter & $26 \mathrm{ml}$ Wasser \\
$200 \mathrm{ml}$ Wasser &
\end{tabular}

Beide Mahlzeiten waren in ihrer Nährstoffzusammensetzung (12\% Kohlenhydrate, 15\% Proteine, 73\% Fett), ihrem Energiegehalt $(523 \mathrm{kcal})$ und ihrem Gewicht (456g) identisch. Die flüssige Testmahlzeit wurde mit dem in einer Studie [3] validierten MRI-Kontrastmittel Gd-DOTA $(600 \mu \mathrm{M})$ markiert, um einen hohen Kontrast zwischen Mageninhalt und umgebendem Gewebe zu erreichen. Der mit der festen Testmahlzeit erreichte Kontrast war hingegen genügend hoch, und es musste kein Kontrastmittel eingesetzt werden. Um die Magensaftsekretion zu bestimmen, wurden definierte Volumina der Testmahlzeit in kleine Flaschen gefüllt, welche als externe Referenz während den Untersuchungen neben die Probanden gelegt wurden.

Untersuchungsprotokoll: Die Probanden wurden in einem 1.5 T Philips ACS NT Tomographen während 120 Minuten nach Verabreichung einer festen oder flüssigen Testmahlzeit untersucht. Das aktuelle Volumen des Mageninhalts wurde (bei der festen und der flüssigen Testmahlzeit) alle 15 Minuten mit einer MultisliceSequenz (28 Schichten, transversale Schichtorientierung, Turbo Spin Echo Sequenz, TE $=40 \mathrm{~ms}, \mathrm{TR}=526 \mathrm{~ms}$, Flip Winkel: $90^{\circ}, 256 * 256$ Pixel, Auflösung: $1.56 \mathrm{~mm}$, Schichtdicke: $7.5 \mathrm{~mm}$ ) erfasst. Bei der flüssigen Testmahlzeit bestand ein linearer Zusammenhang zwischen der Bildintensität des Mageninhaltes und der Magensaftkonzentration. Um auch bei der festen Testmahlzeit eine lineare Abhängigkeit zu erreichen, wurde jeweils eine zusätzliche Messung (Transversale Schichtorientierung, Multislice Turbo Spin Echo Sequenz, 15 Schichten, TE $=12 \mathrm{~ms}$, TR = $100 \mathrm{~ms}$, Flip Winkel: $90^{\circ}, 256 * 256$ Pixel, Auflösung: $1.56 \mathrm{~mm}$, Schichtdicke: $7.5 \mathrm{~mm}$ ) durchgefuihrt. Unmittelbar nach der Volumenmessung und der Sekretionsmessung wurde eine dynamische Messung (Koronale Schichtorientierung, FFE Sequenz, 120 Bilder, zeitliche Auflösung: $1 \mathrm{~s}, \mathrm{TE}=6 \mathrm{~ms}, \mathrm{TR}=13 \mathrm{~ms}$, Flip Winkel: $25^{\circ}, 128 * 128$ Pixel, Auflösung: $3.1 \mathrm{~mm}$, Schichtdicke: $10 \mathrm{~mm}$ ) durchgeführt, mit welcher die Magenmotilität erfasst wurde.

Daten-Analyse: Entleerung: Die Region des Mageninhalts wurde in allen Bildern mit einem halbautomatischen Konturverfolgungs-Algorithmus detektiert. Zur Volumenberechnung des Mageninhalts wurde in jedem Bild der Volumenmessung die Fläche des Mageninhaltes berechnet, und anschliessend wurden alle Flächen summiert und mit der Schichtdicke multipliziert.

Alle berechneten Volumina der 120 minütigen Untersuchung wurden danach in ein exponentielles Enleerungsmodell gefittet und die Halbwertszeit der Entleerung ( $\mathrm{T} 1 / 2[\mathrm{~min}])$ wurde berechnet.

Motilität: Die Motilitätsparameter wurden wie folgt berechnet. In allen Bildern der Motilitätsmessung wurde der Magendurchmesser entlang einer Magenachse berechnet. Basierend auf dieser Durchmesserberechnung wurden anschliessend die Magenkontraktionen detektiert, und die Amplitude ([\%]; 0\% = keine Kontraktion, 100\% $=$ vollständige Einschnürung), die Ausbreitungsgeschwindigkeit $(\mathrm{cm} / \mathrm{s})$ und die Frequenz $\left(\mathrm{min}^{-1}\right)$ der 
Kontraktionen berechnet ( $x \pm$ SEM). Die Auswertungssoftware wurde in $C$ unter Verwendung der Xlib/Motif Bibliothek auf einer DEC Alpha/AXP entwickelt.

\section{ERGEBNISSE}

Figur 1 zeigt zwei Bilder einer Motilitätsmessung nach Verabreichung der festen (links) und der flüssigen (rechts) Testmahlzeit.
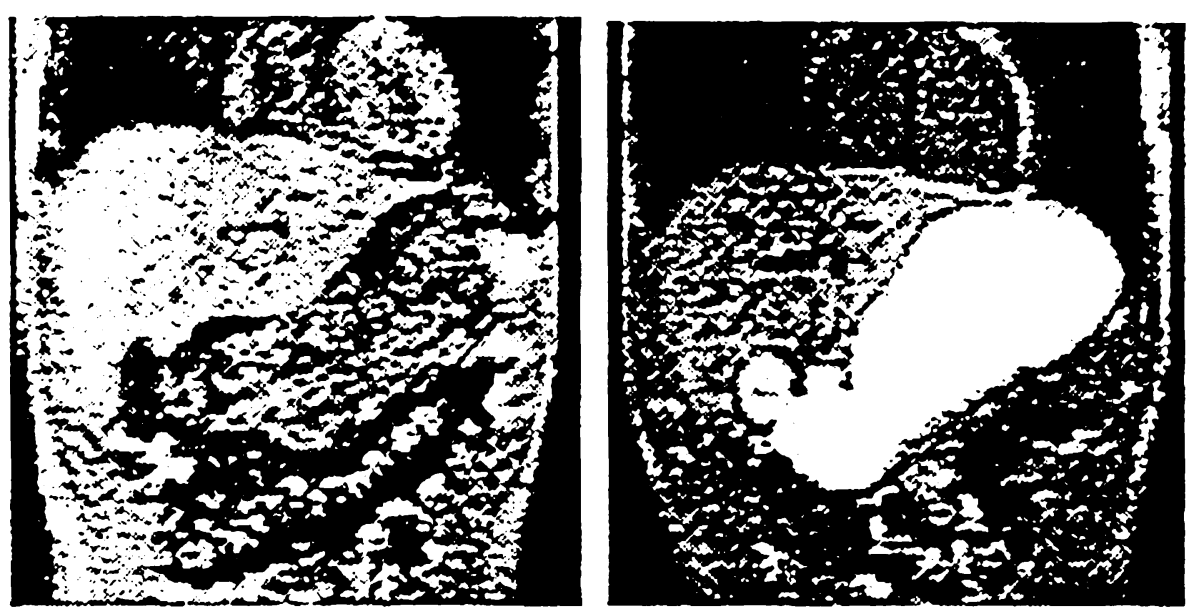

Figur 1: Messung der Magenmotilität: Koronale Schichten nach Verabreichung der festen (links) und der flüssigen (rechts) Testmahlzeit.

Der Mageninhalt und die Magenkontraktionen sind in beiden Bildern deutlich sichtbar. Der hohe Kontrast zwischen Mageninhalt und umliegendem Gewebe erlaubt bei den Bildern der Motilitätsmessung und der Volumenmessung (Figur 2) eine einfache Detektion des Mageninhaltes.
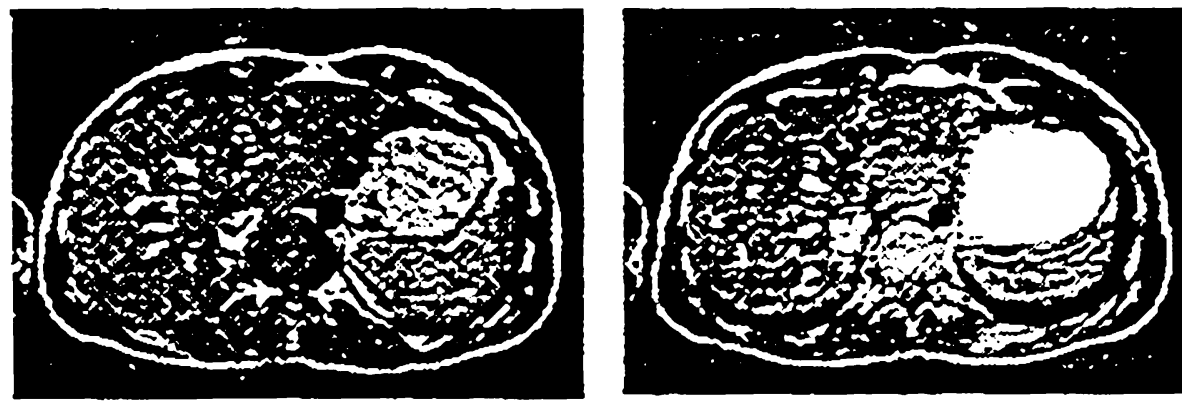

Figur 2: Erfassung der Magenentleerung: Transversale Schichten nach Verabreichung der festen (links) und der flïssigen (rechts) Testmahlzeit.

Ein Ausschnitt des dynamischen Ablaufs einer Motilitätsmessung ist in Figur 3 dargestellt. Die Bewegung der Magenkontraktionen, welche ihren Ursprung im Korpusbereich des Magen haben sind deutlich sichtbar $(\rightarrow)$.

Die Magenentleerung der flüssigen Mahlzeit war signifikant schneller als die Entleerung der festen Mahlzeit $\left(T^{1} / 2\right.$ (flüssig) $=97 \pm 7 \mathrm{~min} ; T^{2} / 2$ (fest) $=127 \pm 9$ min; $p<0.05$ ). In Figur 4 ist die schnellere Entleerung der flüssigen Mahlzeit deutlich in den Entleerungskurven sichtbar. Während der Verarbeitung der flüssigen Mahlzeit wurden antrale Kontraktionen mit höherer Amplitude $(25.3 \pm 1.4 \%)$ beobachtet als bei der festen Mahlzeit $(20.7 \pm 1.0 \% ; p<0.001)$. Die Frequenz (fest: $2.9 \pm 0.1 \mathrm{~cm}^{-1}$; flüssig: $2.8 \pm 0.1 \mathrm{~cm}^{-1}$ ) und die Ausbreitungsgeschwindigkeit (fest: $0.3 \pm 0.1 \mathrm{~cm} / \mathrm{s}$; flüssig: $0.3 \pm 0.1 \mathrm{~cm} / \mathrm{s}$ ) der Kontraktionen waren hingegen nicht unterschiedlich.
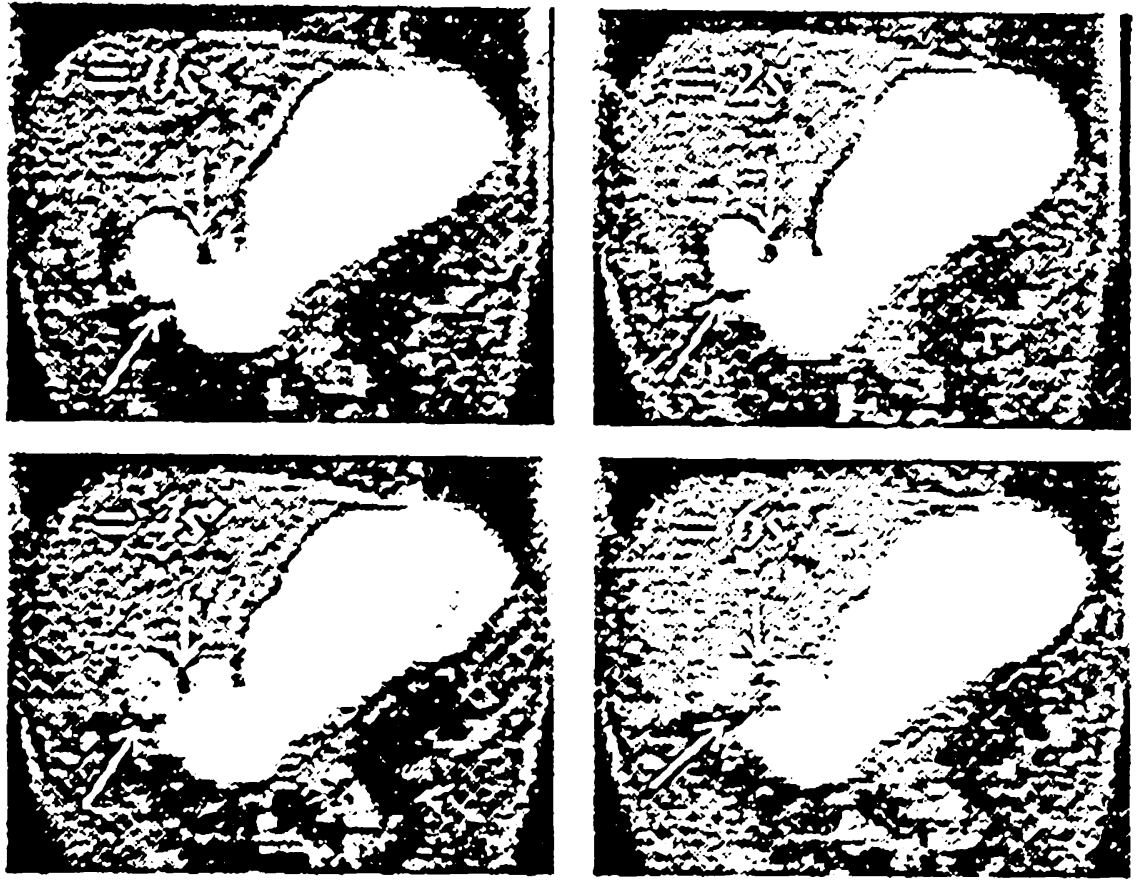

Figur 3: Messung der Magenmotilität: Vier Bilder einer dynamischen Messung (flüssige Testmahlzeit).

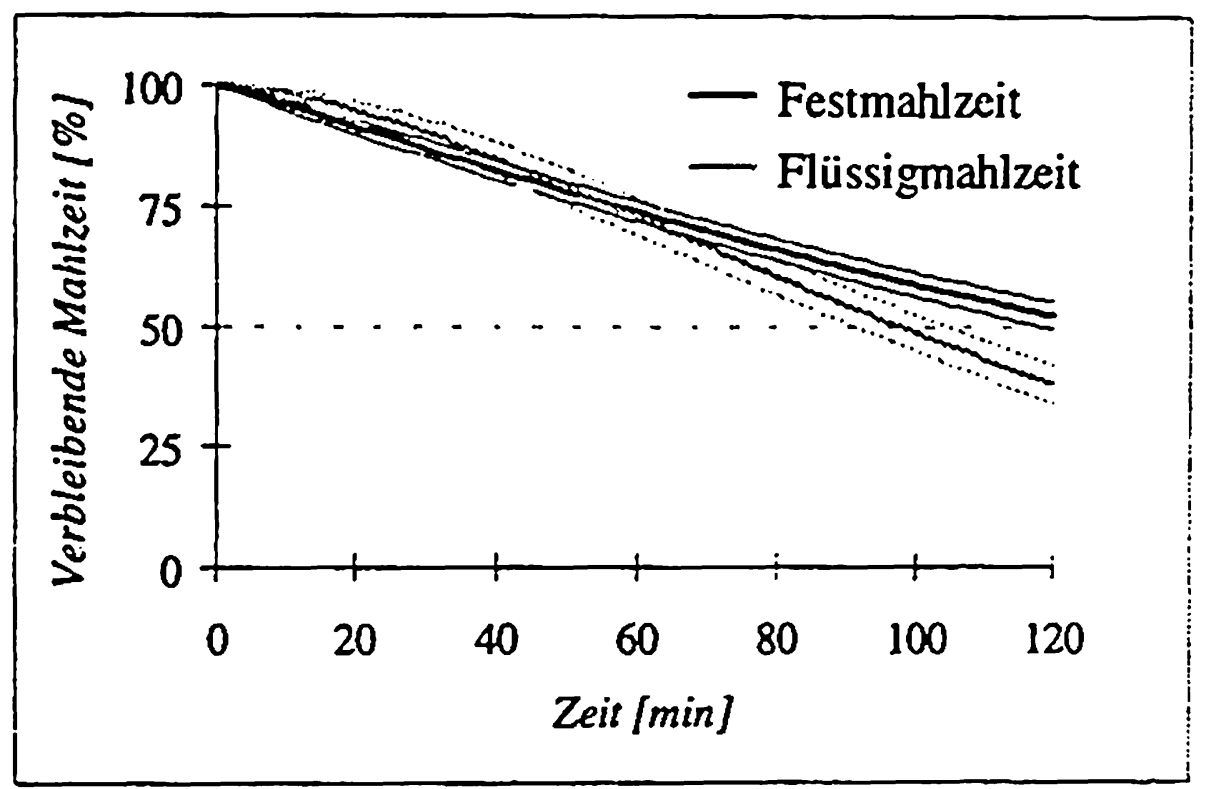

Figur 4: Mittlere Entleerungskurven ( \pm SEM) der festen und der flüssigen Testmahlzeit.

\section{SCHLUSSFOLGERUNGEN}

Mit unserer Studie konnten wir erstmals zeigen, dass es mit bildgebender Magnetresonanz möglich ist, ohne Verwendung eines Kontrastmittels die Magenentleerung und -motilität einer festen Mahlzeit zuverlässig zu erfassen. Im Gegensatz zur vorherrschenden Meinung scheint das Antrum eine wichtige Rolle bei der Regulation der Entleerung flüssiger und fester Mahlzeiten zu spielen, indem die Amplitude, aber nicht die Frequenz und nicht die Geschwindigkeit der antralen Magenkontraktionen variiert werden. Nach der Verabreichung der festen Mahlzeit wurden Kontraktionen mit einer geringeren Einschnürtiefe beobachtet.

\section{LITERATUR}

[1] W. Schwizer, H. Maecke, M. Fried, Gastroenterology 103, 369, 1992.

[2] P. Kunz, P. Boesiger, et al, 2nd ann. meeting of the SMR, 1526, 1994.

[3] W. Schwizer, M. Fried, et al, MRM 31, 388, 1994. 